\title{
SOBRE EL REGRESO DE CARROLL, EL CONVENCIONALISMO Y LOS FUNDAMENTOS DE LA LÓGICA*
}

\section{On Carroll's Regress, Conventionalism and the Foundations of Logic}

\author{
MARIo Gómez-TorRente ${ }^{a}$ \\ https://orcid.org/0000-0002-5957-6544 \\ mariogt@unam.mx \\ ${ }^{a}$ Instituto de Investigaciones Filosóficas, UNAM, Ciudad de México, México
}

\section{Resumen}

Propongo que el argumento carrolliano de Quine en "Truth by Convention" no refuta una variedad especialmente genuina de convencionalismo acerca de la lógica. También argumento que la lección carrolliana básica acerca del convencionalismo es que la introducción de convenciones o tesis aceptadas acerca de qué es lógicamente correcto no puede por sí sola instaurar las disposiciones inferenciales correspondientes. Apoyándome en mi discusión del regreso carrolliano, indico finalmente que hay cuando menos ciertos límites carrollianos a la manera en que las "leyes lógicas" podrían ser "sustituidas" y, en contra de ciertas consideraciones de Alberto Moretti, que hay también otros límites no estrictamente carrollianos a la sustituibilidad de las leyes lógicas.

Palabras clave: Convencionalismo; Adopción de reglas; Seguimiento de reglas; Inferencialismo.

\begin{abstract}
I propose that Quine's Carrollian argument in "Truth by Convention" does not refute an especially genuine variety of conventionalism about logic. I also argue that the basic Carrollian lesson about conventionalism is that the introduction of accepted conventions or theses about what is logically correct cannot by itself create the corresponding inferential dispositions. Based on my discussion of the Carrollian regress, I finally indicate that there are at least certain Carrollian limits to the

*Agradezco sus útiles comentarios sobre estas ideas a auditorios de la UNAM, de la Universidad de Konstanz, de la Universidad de Turín, y del XXIII Congreso Mundial de Filosofía celebrado en Atenas. La investigación recibió apoyo de una beca para una estancia sabática de la DGAPA-UNAM, de un proyecto del CONACyT de México (CCB 2011 166502), del proyecto PAPIIT-UNAM IA-401015, y del Ministerio de Economía, Industria y Competitividad español (proyecto de investigación FFI2015-70707-P).
\end{abstract}


way in which the "logical laws" could be "replaced" and, contrary to some of Alberto Moretti's considerations, that there are also other not strictly Carrollian limits to the replaceability of logical laws.

Key words: Conventionalism; Adoption of rules; Rule-following; Inferentialism.

\section{Introducción}

Pocos dudan, creo, que el regreso en el que la astuta Tortuga de Lewis Carroll (1895) embarca a su opresivo compañero de paradojas, Aquiles, muestra algo (o varias cosas) de importancia para los fundamentos de la lógica (entendidos éstos en sentido amplio, abarcando aspectos metafísicos, epistemológicos y posiblemente otros). Creo que también se puede decir que en general no hay mucha claridad acerca de qué muestra exactamente ese regreso, pero asimismo que el célebre argumento particular de Quine contra el convencionalismo en lógica, inspirado en ese regreso, se considera casi universalmente como una contribución establecida a la discusión sobre los fundamentos de la lógica, y específicamente como una refutación irrebatible de la tesis convencionalista. En este artículo argumentaré, más o menos por oposición a estas opiniones generalizadas: (1) que el argumento carrolliano de Quine no refuta una cierta variedad de convencionalismo acerca de la lógica que, en mi opinión, es seguramente la variedad más genuina de convencionalismo, $\mathrm{o}$ al menos la mejor motivada desde la perspectiva que anima las tesis convencionalistas; y (2) que la lección básica acerca del convencionalismo que se puede extraer del regreso de Carroll es relativamente clara, y consiste en que la introducción de convenciones o tesis aceptadas acerca de qué es lógicamente correcto no puede por sí sola instaurar las disposiciones inferenciales correspondientes-y en particular no puede hacerlo en alguien que no tenga ya esas disposiciones. Algo parecido a esta lección básica ha sido enfatizada por Kripke y sus expositores en años recientes, pero creo que la conexión entre las consideraciones de Kripke y las de Quine es al menos en cierto aspecto diferente de la que el propio Kripke parece haber supuesto, y, en general, que las implicaciones del regreso carrolliano para la filosofía de Quine no se han visto con excesiva claridad; el presente artículo busca también clarificar aunque sea muy parcialmente esas implicaciones.

A lo largo de su carrera, Alberto Moretti ha reflexionado sobre los fundamentos de la lógica con su profundidad y distanciamiento característicos, señalando los pros y, especialmente, los contras de las diversas posturas sobre los aspectos básicos de la epistemología y la metafísica 
de la lógica. ${ }^{1}$ Recientemente Moretti ha abandonado lo que siempre me pareció ser su distintiva actitud neutral y distanciada para proponer una concepción de los fundamentos de la lógica "de aspecto kantiano" (Moretti 2016, 21) según la cual "tener algún sistema de principios lógicos es constitutivo del discurso y de aquello sobre lo que el discurso versa", pero "en la cual (a diferencia del kantismo tradicional) ningún sistema específico de principios es necesario" (ibídem) y "las leyes y verdades lógicas, que siempre son específicas, aunque no son refutables, son sustituibles" (ibíd., 22). Apoyándome en la discusión del regreso carrolliano que llevaré a cabo en este artículo, indicaré que, como han enfatizado también Kripke y sus expositores, hay cuando menos ciertos límites carrollianos a la manera en que las "leyes lógicas" podrían ser "sustituibles". Y, yendo un poco más allá que Kripke, sugeriré, en contra de ciertas consideraciones de Moretti, que hay también otros límites no estrictamente carrollianos a la sustituibilidad de las leyes lógicas, que se siguen de las razonables hipótesis de que los patrones de inferencia usuales son válidos en virtud de las propiedades de las referencias o contenidos de las constantes lógicas, y de que los seres con las características cognitivas apropiadas (como nosotros) tienen su conducta inferencial relativa a esas constantes adaptada a reflejar las propiedades de sus referencias correspondientes; señalaré, en particular, que un cierto tipo de ilusión de sustituibilidad en el ámbito de las leyes lógicas la puede generar una confusión relacionada con estas hipótesis sobre la que, irónicamente, nos alertó el mismo Quine.

\section{El argumento carrolliano de Quine y el convencionalismo radical}

En "Carnap and Logical Truth", Quine da una formulación sucinta de su argumento carrolliano en contra de la idea de que las verdades lógicas son verdaderas por convención:

Logical truths, being infinite in number, must be given by general conventions rather than singly; and logic is needed then to begin with, in the metatheory, in order to apply the general conventions to individual cases. (Quine 1954, 108)

La idea de Quine es que el número de las verdades lógicas es infinito de hecho, y un convencionalista sólo podría sostener que sus

${ }^{1}$ Véase especialmente Moretti (2010). 
convenciones han hecho que este número infinito de verdades lógicas sean verdades si acepta además que las convenciones operan dentro del marco de alguna teoría previamente determinada como verdadera, determinando en ese marco que es un hecho que todas las verdades lógicas en ese conjunto infinito se derivan de las convenciones. Pero este marco metateórico debería entonces ser verdadero por convención desde el punto de vista del convencionalista. Y esto nos embarca en un regreso al infinito de reminiscencias carrollianas, como queda expresado de forma quizá algo más clara en el siguiente pasaje clave del temprano clásico quineano "Truth by Convention":

In the adoption of the very conventions ... whereby logic itself is set up, ..., a difficulty remains to be faced. Each of these conventions is general, announcing the truth of every one of an infinity of statements conforming to a certain description; derivation of the truth of any specific statement from the general convention thus requires a logical inference, and this involves us in an infinite regress. (Quine 1935, 96)

La idea es que la derivación de la verdad de cualquier verdad lógica específica $\mathrm{V}$ a partir de las convenciones generales iniciales $\mathrm{C}$ y (posiblemente) otras verdades requiere la verdad de una nueva ley lógica $\mathrm{L}$, que ha de ser verdadera por convención. Pero la derivación de la verdad de $\mathrm{V}$ a partir de $\mathrm{C}$, otras verdades y $\mathrm{L}$ requiere a su vez la verdad de una nueva ley lógica L', que ha de ser verdadera por convención... y ya estamos embarcados en el regreso. Por ejemplo, la derivación de “ $2+2=4$ o $2+2 \neq 4$ ” es verdadera' a partir de la convención 'Todas las oraciones de la forma "p o no p" son verdaderas' (más la verdad " $2+2=4$ o $2+2 \neq 4$ " es de la forma "p o no $p$ ") requiere la verdad de la ley lógica "Si todas las oraciones de la forma "p o no p" son verdaderas y “ $2+2=4$ o $2+2 \neq 4$ " es de la forma "p o no p", entonces " $2+2=4$ o $2+2 \neq 4$ " es verdadera', que ha de ser verdadera por convención. Pero la derivación de “" $2+2=4$ o $2+2 \neq 4$ " es verdadera' a partir de 'Todas las oraciones de la forma "p o no p" son verdaderas', “ $2+2=4$ o $2+2 \neq 4$ " es de la forma "p o no p", y 'Si todas las oraciones de la forma "p o no p" son verdaderas y " $2+2=4$ o $2+2 \neq 4$ " es de la forma "p o no p", entonces " $2+2=4$ o $2+2 \neq 4$ " es verdadera' requiere a su vez la verdad de la ley lógica 'Si todas las oraciones de la forma " $p$ o no p" son verdaderas, " $2+2=4$ o $2+2 \neq 4$ " es de la forma "p o no p", y si todas las oraciones de la forma "p o no p" son verdaderas y " $2+2=4$ o $2+2 \neq 4$ " es de la forma "p o no p", entonces " $2+2=4$ o $2+2 \neq 4$ " es verdadera, entonces " $2+2=4$ o $2+2 \neq 4$ " es verdadera', que ha de ser verdadera por convención. Etc. 
La primera observación que quiero hacer es que este argumento no afecta a una postura convencionalista acerca de los fundamentos de la lógica que, en mi opinión, constituye una variedad especialmente pura, o exenta de aditamentos no esenciales a la perspectiva que cabe conjeturar motiva las tesis convencionalistas. Llamemos convencionalismo radical acerca de los fundamentos de la lógica a la tesis metafísico-epistemológica de que no hay hechos acerca de qué es lógicamente verdadero o qué se sigue lógicamente de qué que se den o sean cognoscibles independientemente del establecimiento de convenciones lingüísticas. Según esta tesis, las verdades lógicas son verdaderas y los argumentos lógicamente correctos son correctos en virtud de la existencia de convenciones correspondientes; y las verdades lógicas son cognoscibles (a priori) como verdaderas y los argumentos lógicamente correctos son cognoscibles (a priori) como correctos por parte de un sujeto epistémico en virtud de su participación en esas convenciones. Por ejemplo, una de esas convenciones sería la convención de que ' $2+2=4$ o $2+2 \neq 4$ ' es (lógicamente) verdadera, o la de que 'Todos los hombres son mortales' se sigue (lógicamente) de 'Todos los hombres son griegos' y 'Todos los griegos son mortales'.

Naturalmente, el convencionalismo radical, como cualquier otro tipo sensato de convencionalismo (incluidas las doctrinas contra las que va dirigido el argumento de Quine), no es una tesis histórica, sino una tesis acerca del "contexto de justificación". El convencionalista radical no sostiene que alguien en algún momento (aparte quizá de algún filósofo desocupado) ha estipulado las convenciones de las que habla, sino meramente que al hacerlo uno podría en principio obtener una justificación (a priori) para usar asertivamente las oraciones que uno toma como verdades lógicas y para aceptar los argumentos que uno toma como lógicamente correctos.

Pues bien, incluso si otras especies de convencionalista pueden no ser capaces de contrarrestar el desafío carrolliano de Quine, el convencionalista radical puede responder:

Sin duda ningún conjunto infinito de oraciones son (lógicamente) verdaderas por convención en ningún momento particular. Pero uno puede adoptar convenciones acerca de qué es (lógicamente) verdadero o qué se sigue (lógicamente) de qué a medida que piensa en verdades lógicas o consecuencias lógicas particulares. Incluso si ningún conjunto infinito de verdades son de hecho verdaderas por convención en ningún momento particular, no hay ningún obstáculo a que un conjunto potencialmente infinito de verdades lógicas y consecuencias 
lógicas sean cognoscibles por convención. Esto es todo lo que requiere la intuición convencionalista de que el carácter lógico de ciertas oraciones y argumentos puede ser explicado por la introducción de ciertas convenciones, y de que la peculiar naturaleza epistémica de esas oraciones y argumentos puede también ser explicada de esa manera.

El convencionalista radical señala que, incluso si la derivación de la verdad de cualquier verdad lógica particular a partir de una convención general requiere o presupone la verdad (lógica) de un conjunto infinito de oraciones, e incluso si la concepción tradicional entiende el conjunto de las verdades lógicas como un conjunto actualmente infinito, no es necesario que en ningún momento particular de tiempo exista una derivación de la verdad de cualquier verdad lógica particular a partir de una convención general, y tampoco se requiere entender al conjunto de las verdades lógicas como un conjunto actualmente infinito. Lo único a lo que se obliga el convencionalista radical es la tesis de que el conjunto de verdades y consecuencias lógicas es potencialmente infinito. ${ }^{2}$

Vale la pena mencionar en este punto algunas analogías entre la posición convencionalista radical y el constructivismo clásico en matemáticas, que ayudan quizá a hacer aquella menos implausible de lo que podría parecer a primera vista. Así como el constructivista sostiene que no hay un conjunto actualmente infinito de verdades matemáticas (ni ningún otro conjunto actualmente infinito, claro está), el convencionalista radical sostiene que no hay un conjunto actualmente infinito de verdades lógicas. Y así como el constructivista brouweriano sostiene que las verdades matemáticas llegan a ser conocidas a medida que son construidas por la mente del matemático ideal, el convencionalista radical sostiene que las verdades lógicas llegan a ser conocidas a medida que son estipuladas como verdaderas por la persona, o la comunidad, que instaura las convenciones correspondientes. (Naturalmente, hay muchos otros aspectos en los que los dos tipos de posiciones, la convencionalista radical y la constructivista, difieren sustancialmente; por ejemplo, es seguramente parte de la posición convencionalista que existe un gran grado de libertad por parte del instaurador de las convenciones a la hora de escoger las oraciones y argumentos estipulados como verdaderas o correctos, mientras que es seguramente parte de la posición brouweriana que lo que la mente matemática ideal puede

\footnotetext{
${ }^{2}$ En Gómez-Torrente (2019) he mencionado también esta respuesta convencionalista al argumento carrolliano de Quine, usando la expresión convencionalismo finitario para referirme a lo que aquí he llamado convencionalismo radical.
} 
construir no es potestad suya en ningún grado comparable. De todos modos, las analogías existentes entre las dos posiciones me parecen acusadas e importantes.)

El convencionalista radical insiste, pues, en que el argumento de Quine presupone que la posición que se está criticando requiere que las convenciones relevantes determinen que en un momento dado de tiempo hay una infinidad de oraciones que son verdades lógicas. Quine observa, correctamente, que esto sólo podría darse en virtud de hechos no convencionales de algún tipo, por ejemplo hechos matemáticos infinitarios acerca de la derivabilidad de las verdades lógicas a partir de axiomas básicos mediante la aplicación de reglas básicas, o acerca de la verdad de un conjunto infinito de axiomas básicos, tout court. Pero el convencionalista radical rechazaría cualquier apelación a tales hechos, y muy comprensiblemente. Sin duda es parte del espíritu que anima la posición convencionalista el rechazar apelaciones a hechos lógicos o matemáticos que no están fundamentados en decisiones o construcciones humanas. ¿Y cómo podría un hecho infinitario estar fundamentado en decisiones o construcciones humanas? El convencionalista radical observa que, al negar la existencia de tales hechos, está haciendo mayor justicia al espíritu del convencionalismo que los convencionalistas del tipo que Quine busca refutar.

Surgen preguntas interesantes desde un punto de vista histórico precisamente cuando uno inquiere quiénes son los convencionalistas a quienes va dirigido el argumento clásico de Quine en "Truth by Convention". Lo más probable parece ser que el principal blanco sea Carnap, a la vista de que Carnap es el principal blanco de las críticas de Quine en sus textos tardíos sobre el convencionalismo en lógica -aunque Carnap no es mencionado explícitamente como autor criticado en "Truth by Convention"-. Quizá el convencionalismo de Carnap incluyó en algún momento el requisito presupuesto por Quine. Por ejemplo, de la exégesis de Ebbs (2011) se sigue que el convencionalismo de Carnap siempre incluyó el requisito infinitista presupuesto por Quine (en obras como Carnap 1937, 1939, 1942 and 1947). Pero Ebbs también argumenta, en esencia, que si bien el convencionalismo de Carnap siempre incluyó ese requisito, el argumento de Quine no funciona como argumento contra el convencionalismo carnapiano, porque Carnap no requirió adicionalmente que los "hechos metalingüísticos" lógicos fueran determinados por convención. En otras palabras: según esta exégesis, Carnap se habría sentido satisfecho con aceptar que (algunas de) las verdades lógicas metateóricas requeridas para determinar que un cierto conjunto infinito de oraciones (las verdades lógicas clásicas, presumiblemente) son 
verdaderas no son verdaderas por convención. No puedo disputar aquí con Ebbs acerca de esta exégesis, pero parece altamente sospechosa. En primer lugar, no es claro por qué alguien con las inclinaciones generales de Carnap habría de haber aceptado que algunas verdades lógicas no son verdaderas por convención. Pero incluso si Carnap sostuvo este tipo de postura, nos quedaría la queja de que esa postura está claramente indisponible para un convencionalista mínimamente intenso, como el convencionalista radical ciertamente lo es.

\section{¿Qué muestra el regreso de Carroll acerca de los fundamentos de la lógica y el convencionalismo?}

De la discusión precedente concluyo que el argumento de Quine contra el convencionalismo en lógica no refuta el convencionalismo radical acerca de las verdades lógicas y los argumentos lógicamente correctos. Creo que esta conclusión es devastadora para el argumento de Quine, pues como he intentado sugerir, es como mínimo poco claro que un convencionalista acerca de las verdades lógicas y los argumentos lógicamente correctos pueda ser más moderado que un convencionalista radical, dado el espíritu que anima al convencionalismo. Sin embargo, esto nos deja con una preocupación inmediata. ¿Acaso no muestra el regreso carrolliano algo importante y general acerca de la lógica y el convencionalismo? Pero si es así, ¿qué es entonces lo que muestra?

En mi opinión, el regreso de Carroll muestra algo importante y general acerca de los fundamentos de la lógica y el convencionalismo, pero el apropiado argumento carrolliano basado en el regreso no es quineano. Pienso también que las conclusiones de los argumentos carrollianos basados en el regreso que me parecen más importantes y significativos no muestran estrictamente hablando que el convencionalismo radical $u$ otras formas de convencionalismo (incluida la forma de convencionalismo característica de la filosofía de Quine, de la que hablaremos luego) sean falsas. Sin embargo, un aspecto importante de estos argumentos es que muestran que la introducción de convenciones o tesis aceptadas acerca de qué es lógicamente correcto no puede por sí sola instaurar las disposiciones inferenciales correspondientes-y en particular no puede hacerlo en alguien que no tenga ya esas disposiciones. La introducción de convenciones o tesis aceptadas acerca de qué es lógicamente correcto no tiene por qué guiar por sí sola en la adopción de oraciones de la misma forma que las postuladas como verdades lógicas por las convenciones, o en la realización de inferencias de la misma forma que los argumentos postulados como lógicamente correctos por las convenciones. 
Recordemos la historia clásica de Carroll (1895) para aquellos lectores que no la tengan fresca en mente. El artículo de Carroll no contiene otra cosa que esta historia, y no extrae de ella ninguna conclusión filosófica explícita. La historia empieza cuando la Tortuga le pide a Aquiles que considere tres proposiciones, que enumero a continuación junto a posibles formalizaciones suyas en un lenguaje lógico de primer orden con identidad:

(A) Cosas que son iguales a la misma cosa son iguales entre sí. $[(\mathrm{x})(\mathrm{y})(\mathrm{z})(\mathrm{x}=\mathrm{z} \& \mathrm{y}=\mathrm{z} \supset \mathrm{x}=\mathrm{y})]$

(B) Los dos lados de este Triángulo son cosas que son iguales a la misma cosa. $[a=c \& b=c]$

(Z) Los dos lados de este Triángulo son iguales entre sí. [a=b]

La Tortuga dice aceptar A y B pero no Z, y le pide a Aquiles que la obligue, lógicamente, a aceptar Z sobre la base de cosas que ella acepta. La Tortuga parece estar dispuesta a aceptar cualquier otra proposición, aparte de Z, que Aquiles le pueda presentar y que enuncie verdades de la lógica en algún sentido.

Aquiles empieza pidiéndole que acepte

(C) Si A y B son verdaderas, Z ha de ser verdadera.

(Nótese que $(\mathrm{C})$ dice que $\mathrm{Z}$ es una consecuencia necesaria, o quizá simplemente lógica (si aceptamos la identidad entre consecuencia lógica y consecuencia necesaria) de A y B.) Y la Tortuga acepta (C). Pero eso la deja indiferente. La Tortuga también está dispuesta a aceptar

(D) Si A y B y C son verdaderas, $\mathrm{Z}$ ha de ser verdadera

(que dice que $\mathrm{Z}$ es una consecuencia necesaria de $\mathrm{A}, \mathrm{B}$ y $\mathrm{C}$ ). Pero eso la sigue dejando indiferente, y el regreso ya se ha puesto en marcha.

He aquí un argumento carrolliano que busca hacer explícita una limitación del convencionalismo acerca de la lógica:

- Supongamos que alguien acepta un cierto conjunto de proposiciones, $\mathrm{P}_{1}$ a $\mathrm{P}_{\mathrm{n}}$, ya sea por convención o no; y ese alguien acepta también, por convención, que si $\mathrm{P}_{1}$ a $\mathrm{P}_{\mathrm{n}}$ son verdaderas, 
entonces $\mathrm{C}$ ha de ser verdadera, o que todos los argumentos de la forma de $\mathrm{P}_{1}$ a $\mathrm{P}_{\mathrm{n}} / \mathrm{C}$ son argumentos donde se preserva la verdad de las premisas en la conclusión.

- El hecho importante es entonces que esta aceptación por sí sola no tiene por qué obligar a esa persona a aceptar C; pues esa persona no tiene por qué saber cómo inferir $\mathrm{C}$ a partir de $\mathrm{P}_{1}$ a $\mathrm{P}_{\mathrm{n}} \mathrm{y}$ las convenciones si éstas contienen terminología lógica; y no importa cuántas convenciones adicionales que contengan terminología lógica acepte, eso por sí solo no tendrá por qué obligar a esa persona a aceptar C.

- Si esa persona acepta C, lo hará en virtud de una compulsión generada no por su aceptación de las convenciones en cuestión, sino de otra forma.

Así pues, el argumento concluye que una convención que contenga terminología lógica, o, de hecho, una serie de convenciones que contengan terminología lógica, no puede por sí misma proporcionar una base justificatoria para inferir de maneras lógicas, en el sentido de una base que guíe a la hora de inferir lógicamente. Aquiles le presenta a la Tortuga todas las convenciones que se le ocurre que podrían forzarla lógicamente a aceptar $\mathrm{C}$, pero para extraer $\mathrm{C}$ justificadamente de esas convenciones la Tortuga debería ir más allá de lo que las convenciones por sí solas autorizan: la Tortuga tendría que saber cómo inferir a partir de las convenciones mismas en virtud de la terminología lógica que éstas contienen.

Una posible objeción al argumento se basa en la idea de que la Tortuga no entiende lo que está haciendo si dice aceptar (A), (B) y (C) pero no aceptar (Z): la Tortuga debe de carecer de una comprensión apropiada de (A), (B), (C) o (Z). De manera similar-así continuaría la objeción-si alguien acepta que $\mathrm{P}_{1}$ a $\mathrm{P}_{\mathrm{n}}$ son verdaderas y que si $\mathrm{P}_{1}$ a $\mathrm{P}_{\mathrm{n}}$ son verdaderas, entonces $\mathrm{C}$ ha de ser verdadera, o que todos los argumentos de la forma de $\mathrm{P}_{1}$ a $\mathrm{P}_{\mathrm{n}} / \mathrm{C}$ preservan la verdad de las premisas en la conclusión, ese alguien debe estar dispuesto a aceptar $\mathrm{C}$, so pena de no entender a qué se está comprometiendo.

Pero la fuerza de la objeción es dudosa. ¿Por qué habría de saber esa persona cómo inferir a partir de cada convención que le presentan antes de que le digan cómo hacerlo? Para saber cómo inferir a partir de una convención, Carroll nos sugiere, uno necesita otra, pero para saber cómo inferir a partir de esa convención uno necesita otra, y así sucesivamente. Hemos de imaginar a la persona, o a la humanizada Tortuga, como alguien completamente inocente en materia de lógica, como alguien para quien las convenciones que le puedan inculcar son la 
única fuente de información sobre qué vale o no lógicamente. Y si así lo hacemos, comprenderemos que esa persona, o la Tortuga, no se verá bajo ninguna compulsión lógica de inferir nada en particular a partir de las convenciones recién introducidas si éstas contienen terminología lógica.

Así, nuestro argumento carrolliano parece mostrar que uno no puede obtener una guía para inferir de una manera determinada meramente en virtud de su aceptación de convenciones. Uno no puede (ni siquiera en principio, o en el "contexto de justificación") derivar su justificación, en casos en los que intuitivamente debería obtenerse de una forma "lógica", meramente en virtud de su aceptación de ciertas convenciones. Aunque sin duda es verdad que uno puede introducir verdades lógicas o consecuencias lógicas por convención, nuestro argumento parece mostrar que uno no puede "seguir una convención por convención". De una manera algo extraña estamos atorados en nuestras maneras de inferir, en el sentido de que no podemos cambiarlas (completamente) por convención.

Recordemos ahora que el convencionalismo radical es la idea de que no hay hechos acerca de lo que es lógicamente verdadero o acerca de qué se sigue lógicamente de qué que se den o sean cognoscibles independientemente de convenciones lingüísticas. Creo entonces que el convencionalismo radical no es refutado estrictamente hablando por nuestro argumento carrolliano, pero también que el argumento muestra un cierto tipo de limitación del convencionalismo radical: éste no parece poder dar cabida a la idea intuitiva de que nuestras disposiciones inferenciales lógicas habituales dan pie a casos de saber o conocimiento. Al fin y al cabo, el argumento muestra que hay una imposibilidad de un cierto tipo en el supuesto de que nuestras inferencias lógicas son justificables en virtud de convenciones. $\mathrm{Y}$ el convencionalismo radical debe por tanto rechazar aquella idea intuitiva, debe convertirse en una teoría que al menos parcialmente atribuye un error a nuestra concepción preteórica de cuándo se da el conocimiento lógico. El convencionalismo radical da pie a una laguna explicativa en el ámbito de la epistemología de la lógica.

\section{¿Qué muestra nuestro argumento carrolliano acerca del celebrado convencionalismo de Quine?}

A diferencia del convencionalismo radical (y también, probablemente, del convencionalismo carnapiano), el convencionalismo característico de Quine rechaza la idea de justificación fundacionista en favor de una forma de holismo. En particular, se rechaza la idea de justifica- 
ción en cuanto que proporcionada por elementos que guían la adopción de creencias particulares o la realización de inferencias particulares. Pero aun así Quine es un convencionalista en sentido amplio, pues, como el carnapiano y otros, enfatiza que en el fondo tenemos libertad para organizar nuestro conocimiento de muchas maneras alternativas -que no estamos atenazados, por ejemplo, por una subyacente estructura inevitable como las postuladas por Kant o el Wittgenstein del Tractatus-. En particular, tenemos libertad lógica. Quine ha subrayado que incluso las partes más básicas de nuestra lógica clásica pueden ser rechazadas algún día en beneficio de leyes y postulados lógicos diferentes que ofrezcan un mayor grado de coherencia a nuestro sistema global de creencias tal como se basa en el conjunto de datos empíricos que hemos recopilado como especie.

Observemos que el convencionalista quineano se siente cómodo con la idea de que uno no puede obtener una justificación para "sus" verdades lógicas o "sus" consecuencias lógicas meramente en virtud de su aceptación de convenciones apropiadas como las propuestas por el convencionalista radical. Si la justificación es holística, entonces no importa si convenimos que ciertas oraciones van a ser (lógicamente) verdaderas o que ciertos argumentos van a ser ejemplos de consecuencia (lógica), en el sentido de que estas actividades no podrán en último término proporcionar el tipo de interconexiones con nuestro sistema de creencias y con nuestro acopio de datos empíricos en las que debería consistir la justificación requerida. Y el convencionalista quineano presumiblemente se siente cómodo también con la idea de que no son meramente convenciones lo que determina metafísicamente el conjunto de las verdades lógicas; en todo caso este conjunto ha de venir determinado por axiomas y reglas que no se pueden introducir meramente por convención. Pero insistamos: para Quine la lógica es convencional en el sentido de que hay cierta libertad para escoger la que queramos dentro de ciertos límites (incluso si nuestra elección de una lógica no es lo que nos da una justificación para creer sus verdades lógicas particulares, o lo que determina su conjunto de verdades lógicas).

Ahora bien, aunque el convencionalismo quineano es por tanto relativamente débil tanto epistemológica como metafísicamente en cuanto comparado con el convencionalismo radical, nuestro argumento apunta de nuevo a una dificultad epistemológica en su seno, en este caso algo que podríamos calificar como una especiosidad. Pues incluso si, como sugiere Quine, tenemos una amplia libertad para escoger una lógica, e incluso si no es esta capacidad de escoger lo que nos proporciona una justificación para aceptar ciertas verdades lógicas o lo que por sí 
mismo convierte en un hecho que sean verdades lógicas, en principio debemos aceptar las consecuencias de nuestra lógica elegida infiriendo lógicamente a partir de nuestras convenciones. $\mathrm{Y}$ si nuestro argumento de más arriba es correcto, nuestras nuevas convenciones no nos guiarán sobre cómo hacer esto-inferiremos, si es que lo hacemos, de la manera en que siempre lo hemos hecho. Así, sólo puede ser en un sentido algo especioso que podemos "escoger" una lógica: incluso si podemos elegir ver qué podemos "hacer" con ciertos principios de nuestra elección, la forma en que nos comportaremos inferencialmente, por así decir, no se regirá en ningún sentido apropiado por esos principios.

¿Podría objetar el quineano que él puede al menos escoger las verdades lógicas y las consecuencias lógicas que quiera a medida que las vaya considerando? De esta manera, respondería a nuestro nuevo argumento carrolliano de una manera similar a la que vimos que el convencionalista radical usaba para responder a la carrolliana objeción quineana de "Truth by Convention". Pero observemos que el convencionalista radical decía esto porque de esta manera mostraba cómo podría entenderse que las verdades lógicas se pueden conocer una por una, por convención, en el "contexto de justificación". En contraste con esto, dada su concepción holística de la verdad y la justificación, para Quine el conjunto entero de las verdades lógicas escogidas deben ser justificadas, o "enfrentarse al tribunal de la experiencia", juntas. ¿Qué podría determinar qué conjunto es este, aparte de su derivabilidad, por medio de las reglas lógicas de siempre, a partir de las convenciones? Si no hay respuesta a esta pregunta, entonces el convencionalismo quineano no puede evadir la fuerza del nuevo argumento carrolliano más que en un sentido especioso.

Según Berger (2011) y Padró (2015, cap. 4), Kripke reprocha a Quine el haber mantenido la tesis de que la lógica se puede identificar con un conjunto de verdades, las verdades lógicas de nuestro sistema global de creencias, sin percatarse de que, irónicamente, su argumento carrolliano de "Truth by Convention" y "Carnap and Logical Truth" excluye precisamente la posibilidad de que la lógica se pueda identificar con un conjunto de verdades. La idea de Kripke parece ser que el argumento carrolliano de Quine mostraría que la lógica, si ha de cumplir su papel de codificar la permisibilidad o la obligación de inferir unas verdades a partir de otras, ha de consistir en algo más que verdades, en contra de las afirmaciones explícitas de Quine. Desde mi punto de vista, por el contrario, el argumento carrolliano de Quine se aplica incluso bajo la hipótesis de que la lógica consiste, por ejemplo, en un conjunto de verdades más un conjunto de reglas de carácter permisivo o imperativo. Lo 
que excluye el argumento es meramente que, si el conjunto de verdades lógicas es infinito, ese conjunto se pueda justificar o constituir exclusivamente en virtud de convenciones, tengan éstas carácter asertivo o no. Pues tanto si una convención tiene carácter asertivo como si no lo tiene, mientras contenga terminología lógica será necesaria (desde el punto de vista del convencionalista) otra convención para extraer consecuencias de ella. Quine podría haber aceptado, en contra de sus afirmaciones explícitas, que la lógica es algo más que un conjunto de verdades, y aun así mantener la validez de su argumento carrolliano. ${ }^{3}$ Sin embargo, aunque creo entonces que el argumento carrolliano de Quine no es incompatible en un sentido profundo con su filosofía y en particular con su concepción básica de la constitución de la lógica dentro de nuestro sistema global de creencias, creo también que nuestro argumento carrolliano explicitado más arriba muestra la existencia de al menos una dificultad epistemológica más profunda en la filosofía de la lógica de Quine. Esta filosofía insiste en la libertad de escoger una lógica, dentro de ciertos límites, claro está. Pero nuestro argumento carrolliano indica que esta libertad, si existe, es considerablemente más limitada de lo que Quine parece dar a entender, y por tanto que hay una considerable especiosidad en la tesis quineana de que tenemos libertad (aun dentro de ciertos límites) para escoger una lógica.

En cualquier caso, Kripke, según sus expositores, ha acusado también a su manera a Quine de una especiosidad similar, como vamos a mencionar a continuación.

\section{Nuestro argumento carrolliano y la cuestión de la "sustituibilidad" de las leyes lógicas}

La discusión de las implicaciones de nuestro argumento carrolliano para la filosofía de Quine ilustra una dificultad general, enfatizada por Kripke y sus expositores (véanse especialmente Berger (2011), Padró (2015) y Stairs (1978), (2006), (2016)), para la idea, tan popular en tiempos recientes y quizá mayoritaria entre los filósofos, de que tenemos libertad para escoger una lógica, de que ni la lógica clásica ni ninguna otra son inamovibles en nuestro sistema de creencias y procedimientos de generación de creencias. La dificultad hallada por Kripke, según sus expositores, es que la fábula carrolliana sugiere claramente que no es posible adoptar una lógica, porque al intentarlo, haciendo firme

\footnotetext{
${ }^{3}$ Padró $(2015,112$ ss.) también ve dificultades a la hora de atribuir al argumento carrolliano de Quine todas las potencialidades que Kripke parece haberle atribuido.
} 
propósito de usar ciertos novedosos esquemas axiomáticos y/o reglas, uno no tendrá más remedio que enunciarlos por medio de proposiciones en algún sentido generales que contengan terminología lógica, a partir de las cuales uno sólo podrá inferir de la manera en que siempre lo ha hecho, en virtud de las reglas que codifican su conducta inferencial preexistente relativa a esa terminología.

La dificultad de Kripke me parece evidentemente real, especialmente porque hay ciertamente poca distancia entre ella y las conclusiones de nuestro argumento carrolliano acerca del convencionalismo en lógica. Al fin y al cabo, ¿de qué forma podría adoptar uno una nueva lógica sino estableciendo lo que en esencia serían convenciones de emplear ciertos novedosos esquemas axiomáticos y/o reglas? Por el mismo motivo por el que esas convenciones no podrían guiar la conducta inferencial de alguien que las hiciera, esa persona no podría realmente adoptar una lógica nueva o sustancialmente nueva. Tanto la dificultad de Kripke como nuestro argumento carrolliano apuntan, pues, a límites bastante severos para la idea de que las leyes lógicas son "sustituibles", una idea que vimos que Moretti ha suscrito en su obra reciente.

Ahora bien, el amigo de la sustituibilidad podría quizá decir que, incluso si Kripke tiene razón y no es posible adoptar una lógica sustancialmente nueva, ha de ser posible sin embargo que las personas desarrollen, de formas impremeditadas o inconscientes, conductas inferenciales en conflicto con las de su lógica anterior. Además, por todo lo que hemos dicho aquí, queda la posibilidad de que diferentes conductas inferenciales, surgidas en virtud de hechos no puramente convencionales, satisfagan el objetivo quineano de organizar nuestro sistema de creencias de manera exitosa. Por todo lo que hemos dicho hasta aquí, podría decir el amigo de la sustituibilidad como ejemplo, es posible que las personas desarrollen, de forma no premeditada, la conducta de inferir de acuerdo con el patrón (falaz según la lógica clásica) de "afirmar el consecuente" (inferir el antecedente de un condicional material a partir del condicional y de su consecuente), y que en virtud de otros cambios adicionales en sus patrones inferenciales y creencias, organicen su sistema global de creencias de manera exitosa.

El amigo de la sustituibilidad habría de intentar prevenirse aquí contra una observación debida, irónicamente, al propio Quine (en su Philosophy of Logic, Quine (1970)). El mero hecho de que la gente empezara a inferir de acuerdo con lo que superficialmente parecería el patrón de "afirmar el consecuente" no implicaría concluyentemente que estuvieran realmente infiriendo de acuerdo con ese patrón. Pues sólo si la conectiva que usaran tuviera realmente el contenido del condicional 
material (que según la concepción semántica referencialista dominante podemos identificar con su función de verdad) podría decirse que estarían infiriendo de acuerdo con un patrón nuevo, falaz según la lógica clásica. El mero hecho de que la gente empezara a inferir de acuerdo con lo que superficialmente parecería el patrón de "afirmar el consecuente" es consistente con la posibilidad de que el contenido de la conectiva que utilizaran en esos casos no fuera ya realmente la función de verdad que asociamos con el condicional material. Quine, en virtud de una serie de aspectos conocidos de su teoría del significado, de hecho sugiere que un cambio en los patrones de inferencia superficiales relativos a una cierta conectiva aparente implicaría directamente que el contenido de esa conectiva cambiaría también. Según la famosa forma de expresarlo de Quine, un cambio de lógica es (o podría ser, diría yo de manera más débil) sólo un cambio de tema o contenido. ${ }^{4}$

Dudo que el amigo de la sustituibilidad pueda prevenirse de manera efectiva contra la observación de Quine. Pues creo que esta observación, y en general la existencia de límites no carrollianos a la sustituibilidad de las leyes lógicas, se siguen de elementos básicos de nuestra concepción intuitiva del contenido o referencia de las expresiones lingüísticas en general y del contenido de las expresiones lógicas en particular, así como de nuestra concepción intuitiva de nuestro vínculo epistémico con esos contenidos. Según la concepción general, una palabra tiene, al menos en el caso estándar, una cosa de un cierto tipo como contenido o referencia, y algunas verdades acerca de esa cosa se reflejan normalmente en los principios acerca del uso de la palabra que los hablantes llegan paulatinamente a aceptar o al menos a observar implícitamente. Así, por ejemplo, un nombre propio usual tiene como referencia a un objeto individual, una cosa que no es susceptible en principio de aplicarse a otras, y esto se refleja en el hecho de que los hablan-

${ }^{4}$ Muchos han reprochado a Quine una inconsistencia entre esta idea y la idea de que es posible cambiar nuestra lógica de modo que nuestro sistema de creeencias sea tanto o más empíricamente adecuado que el actual. (Véase por ejemplo Berger (2011), 188, n. 11, para algunos ejemplos.) Mi punto de vista personal es que no hay necesariamente aquí una inconsistencia en la filosofía de Quine, pues cuando Quine dice que es posible cambiar nuestra lógica no niega que esos cambios conlleven cambios del contenido de la terminología lógica, tal como él entiende la noción de contenido o significado. Recuérdese que la concepción quineana del significado no es la concepción referencialista dominante, sino una concepción holista-inferencialista en la que el significado de una expresión viene dado por el conjunto de principios que los hablantes siguen en su conducta lingüística relativa a esa expresión. Quine mismo ha observado esto en varios lugares, aunque no siempre ha convencido a los que le reprochan una inconsistencia en este ámbito. 
tes observan implícitamente el principio de que un nombre propio no es susceptible en principio de predicarse de otras expresiones. Un nombre común usual tiene como referencia a una propiedad, una cosa que es en principio susceptible de aplicarse a otras, y esto se refleja no sólo en el hecho de que los hablantes observan implícitamente el principio de que un nombre común es susceptible en principio de predicarse de otras expresiones, sino también, a menudo, en el hecho de que los hablantes predicarán normalmente un nombre común de nombres para las cosas a las que se aplica efectivamente la propiedad que es la referencia de ese nombre común, y en el hecho de que predicarán de los nombres para esas cosas propiedades que conlleva la posesión de la propiedad que es la referencia de ese nombre común, o que conlleva la posesión de esa propiedad junto con la posesión de otras propiedades conocidas. Así, por ejemplo, si Juana es hermana de Petra, en casos normales los hablantes predicarán 'es hermana de' de Juana y Petra, y predicarán de ellas 'tienen un progenitor en común'; y si se sabe que Juana tiene como progenitores a José y Luisa, los hablantes predicarán 'tiene como progenitor a José y/o Luisa' de Petra.

Las constantes lógicas no tienen por qué ser una excepción a este fenómeno general. Supongamos que la referencia de la palabra 'si' es la función de verdad del condicional material, que podemos ver como una propiedad que se aplica exactamente a los pares ordenados de proposiciones donde o bien la primera proposición es falsa o la segunda es verdadera. Entonces los hablantes competentes con la palabra 'si' entendida de esta forma predicarán normalmente 'si' de los pares de proposiciones a los que se aplica efectivamente la función de verdad del condicional material, y predicarán de esas proposiciones propiedades que conlleva la posesión de esa función de verdad, o que conlleva la posesión de esa función de verdad junto con la posesión de otras propiedades conocidas. Así, por ejemplo, si $\mathrm{P}$ es falsa o $\mathrm{Q}$ es verdadera, en casos normales los hablantes predicarán 'si' de $\mathrm{P}$ y $\mathrm{Q}$, en ese orden, y predicarán 'no es el caso que ... o _' de $\mathrm{P}$ y $\mathrm{Q}$, en ese orden; y si se sabe que $\mathrm{P}$ no es falsa, sino verdadera, los hablantes predicarán 'es verdadera' de $\mathrm{Q}, \mathrm{o}$, en otras palabras, harán un uso implícito del principio de modus ponens.

Si esto es así, se nos ofrece un argumento bastante razonable tanto para la idea de que un cambio de lógica es sólo un cambio de tema como para la idea más general de la existencia de severos límites no estrictamente carrollianos a la sustituibilidad de las leyes lógicas. Supongamos que los patrones lógicos de inferencia usuales son válidos en virtud de las propiedades de las referencias o contenidos de las constantes lógicas, y que los seres con las características cognitivas apropiadas 
(como nosotros) tienen su conducta inferencial relativa a esas constantes adaptada a reflejar las propiedades de sus referencias correspondientes. Entonces ha de ser difícil que las personas desarrollen, incluso de formas impremeditadas o inconscientes, conductas inferenciales en conflicto con las de su lógica establecida. Pues las conductas inferenciales asociadas a la lógica establecida no son arbitrarias, fundadas en una mera convención o justificadas en virtud de su ajuste global dentro de un gigantesco sistema de creencias. Son conductas asentadas en virtud de que responden a propiedades de las referencias establecidas de la terminología lógica, y de que tales propiedades tienden precisamente a reflejarse y asentarse en la conducta inferencial de los hablantes, presumiblemente en virtud de que la correspondencia con la realidad, de la que la verdad y la inferencia correcta son manifestaciones, tiene un valor de adaptación a las circunstancias del mundo que habitamos. Y entonces la idea de que un cambio de lógica es sólo un cambio de tema resulta también muy razonable, porque en la medida en que un cambio de lógica sea posible, lo más probable es que consista en la adquisición de conductas inferenciales que, incluso cuando sean superficialmente diferentes de las anteriores, reflejen las propiedades de nuevas referencias adquiridas por las constantes lógicas. Pues sólo conductas inferenciales que reflejaran tales propiedades podrían aspirar a tener a la larga un valor suficiente de adaptación a la realidad.

$\mathrm{Al}$ considerar posturas que, como la recién esbozada, atribuyen el fundamento de la verdad lógica a "la estructura de la realidad, presuntamente independiente de las estructuras del lenguaje" (Moretti (2016), 11), Moretti objeta, de manera resumida, que "cuando se [quiere] que todo el contenido de las verdades lógicas [descanse] en leyes del mundo extralingüístico, advertimos que la comprensión de ese mundo parece necesitar verdades lógicas conceptualmente previas" (Moretti (2016), 12). En nuestro caso de más arriba, podríamos decir, la ley extralingüística que fundamenta las inferencias que siguen el principio de modus ponens sería una ley basada en la estructura -matemática, si se quiere-de la función de verdad del condicional material: la propiedad de ser verdadera de una proposición 'si P, Q', junto con la propiedad de ser verdadera de la proposición $\mathrm{P}$, necesita que la proposición $\mathrm{Q}$ tenga la propiedad de ser verdadera. Y esta ley podría recibir una prueba inmediata que utilizara el hecho de que 'si' refiere a una función de verdad que excluye la posibilidad de que 'si P, Q' y P sean verdaderas y $\mathrm{Q}$ no sea verdadera. Utilizando un ejemplo análogo, Moretti se pregunta: 


\begin{abstract}
¿Pero cómo entendemos esa ley formal? ¿Qué significado le atribuimos a la oración que la expresa? Entender una oración supone alguna lógica, porque los principios lógicos son los que permiten la determinación recursiva o composicional de los significados, sin la cual no podemos explicar el intercambio lingüístico. ¿Cómo, entonces, concebir una estructura legal en el mundo sin presuponer una estructura lógico-lingüística? No hay leyes formales si no hay principios lógicos que establezcan relaciones de tipo inferencial entre aquello que está sujeto a esas leyes. Por otra parte, ¿cómo podemos saber que existe aquella ley formal? ¿Cómo saber que la oración que pretende describirla es verdadera? (...) suponer lógica (para entender y concebir leyes formales del mundo) y suponer pruebas (para creer que existen) es suponer la verdad de enunciados y leyes lógicas. Por tanto, esas verdades y esas leyes lógicas supuestas no podrán fundarse en leyes matemáticas, en particular en leyes formales del mundo a las que tengamos acceso previo. (Moretti (2016), 11-12)
\end{abstract}

Moretti, pues, objeta a posturas como la que hemos esbozado en este artículo que tanto entender como demostrar una ley extralingüística como las que fundamentan las inferencias que siguen los principios lógicos presupone "la verdad de enunciados y leyes lógicas".

Supongamos o concedamos que así es. ¿Qué fuerza tiene esta observación contra la idea de que el fundamento de la lógica son las propiedades de las referencias de las constantes lógicas? Creo que muy poca. Entender y demostrar son actividades que involucran el lenguaje de una forma esencial. Y el uso del lenguaje requiere un vasto entramado de presuposiciones, que podemos conceder que incluye de alguna forma más o menos indirecta a las leyes lógicas. Pero ese entramado contiene también presuposiciones de muchos otros tipos que presumiblemente deben su verdad a aspectos de la estructura extralingüística del mundo. Sin ir más lejos, incluye la presuposición de que hay objetos individuales, cosas que no son susceptibles en principio de aplicarse a otras, tal como se refleja en el hecho de que los hablantes usan nombres propios típicos, no susceptibles en principio de predicarse de otras expresiones. O la presuposición de que hay propiedades, cosas que son en principio susceptibles de aplicarse a otras, tal como se refleja en el hecho de que los hablantes usan nombres comunes típicos, susceptibles en principio de predicarse de otras expresiones. Entender y argumentar a favor de estas presuposiciones sin duda presupone de formas más o menos indirectas a esas presuposiciones mismas. ¿Debemos pensar entonces que la verdad de las presuposiciones de que hay objetos individuales y pro- 
piedades es una ilusión, o que en el mejor de los casos no descansa en la existencia extralingüística de entidades correspondientes? Claramente esto sería ir demasiado lejos. En todos estos casos nos las vemos con verdades sobre aspectos tan básicos de la estructura del mundo que no es implausible pensar que nuestro discurso acerca de ellas mismas las presupone de alguna forma más o menos indirecta. Pero esto no es ninguna razón para pensar que son algún tipo de ilusión, sino, muy por el contrario, una poderosa razón para pensar que la estructura de la realidad es responsable de haberlas impuesto paulatinamente en nuestro discurso, en virtud de la capacidad adaptativa que confiere a un discurso su facultad de corresponderse con la realidad. Como mínimo, es esta última una idea más plausible que la dudosa idea antirrealista de que las presuposiciones básicas de nuestro lenguaje son en algún sentido ilegítimas porque son presupuestas también por nuestras actividades cognitivas acerca de ellas.

Si bien dudosas ideas antirrealistas de diversos tipos han hecho pensar a muchos, incluido Moretti, que el fundamento de la lógica no puede hallarse en la realidad extralingüística, y debe hallarse más bien en alguna convención (en sentido amplio), altamente arbitraria y sustituible, lo cierto es que yo no veo razón para pensar que el caso de la lógica sea sustancialmente diferente del de otras clases de verdades que deben su fundamento a la realidad extralingüística, y sí varias razones (algunas de las cuales hemos esbozado en este artículo) para pensar que su fundamento no es convencional (en ninguno de los sentidos de 'convencional' que hemos considerado aquí). Queda la tarea de argumentar de manera más positiva y con mayor detalle que el fundamento de la lógica se halla en ciertos aspectos de la realidad extralingüística. Pero ese será, espero, el objeto de otro trabajo.

\section{Bibliografía}

Berger, A. (2011). Kripke on the incoherency of adopting a logic. En A. Berger (Comp.), Saul Kripke (pp. 177-207). Cambridge University Press.

Carnap, R. (1937). The logical syntax of language. Kegan Paul, Trench, Trubner \& Co.

Carnap, R. (1939). Foundations of logic and mathematics. Encyclopedia of Unified Science, 1 (3).

Carnap, R. (1942). Introduction to semantics. Harvard University Press. Carnap, R. (1947). Meaning and necessity. University of Chicago Press. Carroll, L. (1895). What the tortoise said to Achilles. Mind 4, 278-280. 
Ebbs, G. (2011). Carnap and Quine on truth by convention. Mind 120 (478), 193-237.

Gómez-Torrente, M. (2019). Soames on the logical empiricists on truth, meaning, convention, and logical truth. Philosophical Studies 176, 1357-1365.

Moretti, A. (2010). Concepciones de la lógica. Páginas de Filosofía 11 (13), 162-185.

Moretti, A. (2016). La lógica y la trama de las cosas. Ideas y Valores 65, 5-22.

Padró, R. (2015). What the tortoise said to Kripke: the adoption problem and the epistemology of logic (tesis doctoral). City University of New York.

Quine, W. V. (1935). Truth by convention. En W. V. Quine, The ways of paradox and other essays (1966, pp. 70-99). Harvard University Press.

Quine, W. V. (1954). Carnap and logical truth. En W. V. Quine, The ways of paradox and other essays (1966, pp. 100-125). Harvard University Press.

Quine, W. V. (1970). Philosophy of logic. Prentice-Hall.

Stairs, A. (1978). Quantum mechanics, logic and reality (tesis doctoral). University of Western Ontario.

Stairs, A. (2006). Kriske, Tupman and quantum logic: the quantum logician's conundrum. En W. Demopoulos e I. Pitowsky (Comps.), Physical theory and its interpretation. Essays in honor of Jeffrey $B u b$ (pp. 253-272). Springer.

Stairs, A. (2016). Could logic be empirical?. En J. Chubb, A. Eskandarian y V. Herizanov (Comps.), Logic and algebraic structures in quantum computing (pp. 23-41). Cambridge University Press. 